\section{Illicit drug misuse in mental health units}

Sir: We were interested to read Williams \& Cohen's (Psychiatric Bulletin, February 2000, 24, 43-46) reminder of the difficulties for front-line staff in managing the problem of illicit drug use in mental health units. They suggest that "clear procedures to control substance misuse are necessary ... for the legal protection of staff" in addition to policies covering patient and visitor searches, consultation with local police and "how far can and should confidentiality be protected".

Their comments are pertinent in light of the recent sentencing of two Cambridgeshire hostel workers to four and five years' imprisonment under the 'Premises' section of the Misuse of Drugs Act, which makes it a criminal offence for third parties to knowingly permit heroin or cannabis use in their property, in this case a homelessness day centre (The Guardian, 10 December 1999). Although suspected drug dealers were banned from the centre, staff refusal to give the names of alleged drug users to the police on the basis of confidentiality was seen as 'deliberately obstructive' behaviour.

With the reported prevalence of comorbid psychotic and substance misuse disorders being high and with such patients spending longer in hospital (Menezes et al, 1996), legal issues surrounding the presence of alcohol and drugs in mental health units are bound to occur. It would be detrimental to an already (dually) disadvantaged group of patients if staff felt unsure or even afraid of the legal consequences of their management relating to prohibited substances and we too urge trusts to offer clear guidance for the protection of both patients and their staff.

\section{Reference}

MENEZES, P. R., JOHNSON, S.,THORNICROFT, G., et al (1996) Drug and alcohol problems among individuals with severe mental illness in south London. British Journal of Psychiatry, 168, 612-619.

*John Milton Lecturer in Forensic Mental Health, East Midlands Centre for Forensic Mental Health, Arnold Lodge, Cordelia Close, Leicester LE5 OLE, e-mail: milton@lineone.net, Ira Unell Lecturer in Substance Misuse, Leicestershire NHS Drug and Alcohol Service, Drury House, 50 Leicester Road, Narborough, Leicestershire LE9 5DP

\section{Time for locked drug free psychiatric wards?}

Sir: Illicit drug use is endemic in our society, and therefore also in our hospitals. Psychiatric hospitals look after a particularly vulnerable patient group in which drug misuse complicates management and can lead to accidental death. In the article by Williams \& Cohen (Psychiatric Bulletin, February 2000, 24, 43-46) they point out gaps between hospital policy and practice, in the context of clinical governance. However, I feel this only begins to address one of the fundamental issues. The issue is tolerance of people's lifestyles particularly when an in-patient is held, using a Mental Health Act section, against their will. However, something is wrong if this tolerance puts at risk other patients through the availability of drugs on a ward because the 'culture' is one of drug use among the peer group.

Discharge is not always an option due to the clinical condition of the patient and the element of 'proof' of supply is always a difficult task. At the current time staff struggle on with limited support and develop an increasingly antagonistic attitude to 'drug users'. An accident is waiting to happen, and the hospital trust could be seen as liable.

The options, as I see it, once all patients are screened on admission for illicit drugs in or on them, is that drug users go to the 'open drug' wards. The other patients being put in 'drug-free' wards. Alternatively, if the concept of open drug wards is a step too far, then the patients who would have gone to the open drug wards instead go to a locked drug-free psychiatric ward. However, even in a locked unit it is difficult to keep drugs out, but at least it would protect other patients who need and want to be in a drug-free environment.

Malcolm Bruce Consultant Psychiatrist in Addiction, Community Drug Problem Service, 22-24 Spittal Street, Edinburgh EH3 9DU;

e-mail: malcolm.bruce@talk21.com

\section{Use of the Mental State Examination by psychiatric trainees}

Sir: I agree with Kareem \& Ashby (Psychiatric Bulletin, March 2000, 24, 109-110) that the Mental State Examination (MSE) is fundamental to psychiatric evaluations. The result of their audit showing inadequate recording of the MSE by psychiatric trainees, although the presentation of the data begs a number of questions, is, therefore, a cause for concern.

A "standardised format" is suggested as the solution lest trainees should "employ their discretion" such that "important MSE headings and parameters are often unexamined and unrecorded". The implication is that as long as every box on the audit sheet can be ticked then all will be well with the world. Surely the important thing is the content and quality of the MSE and that it meaningfully relates to the patient's condition at the time. Of course, the form in which this information is set out is relevant, but making an industry out of this is to miss the point. There is, excluding hair-splitting, a well-established convention for recording the MSE and a trainee forgetting to ask about abnormal perceptions (or indeed to examine the nervous system) is down to the trainee and not to the absence of a proforma.

I would also argue that it is selfevidently the responsibility of the consultant, as the educational supervisor of the trainee and the doctor in charge of the patient's care, to review the quality of information in the case notes, including admission-clerking and MSE, as well as admission and discharge summaries and clinic letters. How else is one to know what the standards, strengths and weaknesses of a trainee in these important areas are and, therefore, to be in a position to help them to address any shortcomings and contribute to an improved level of clinical care? The audit process can be useful, but is not an alternative to the fundamentals of good practice or the rigorous clinical teaching of trainees, nor should it have to be a means to this end.

Andrew F. Blakey Consultant Psychiatrist, Ingersley Building, Macclesfield General Hospital, Victoria Road, Macclesfield SK10 3BL

\section{Implications of community treatment orders}

Sir: I should like to comment on some of the points made by Llewellyn-Jones \& Donnelly (Psychiatric Bulletin, March 2000, 24, 16-17) in their letter about community treatment orders (CTOs). First, they minimise the importance of the sideeffects of medication. These are not only extrapyramidal in nature, but encompass a large number of other undesirable symptoms, which many patients, quiet reasonably, do not wish to experience. Their observation that tardive dyskinesia can occur in patients who have never taken medication is a non-sequitur - would they similarly dismiss the role of smoking in causing lung cancer on the grounds that some people who do not smoke also develop the disease?

Second, the suggestion that psychiatrists might have a 'duty' to enable their patients to comply with treatment in the community is a dangerous one, as it implies that in certain circumstances we are 'morally obliged' to go against people's wishes for their own good. This is a familiar argument which has been used to justify various forms of coercive and/or radical treatment (including psychosurgery - see for instance William Sargant's (1967) The Unquiet Mind). No doubt psychiatrists, just as much as doctors in other fields of medicine, would like their patients to comply with the treatment 